\title{
Scioglievolezza, serratezza, sporchevole: dubbi scioglibili scioglievol? Speriamo di riuscire a scioglierli!
}

\author{
Raffaella Setti
}

PUBBLICATO: 19 MARZO 2019

\begin{abstract}
Quesito:
Alcuni lettori ci segnalano i termini scioglievolezza e scioglievole che, diffusi anche grazie a una nota pubblicità televisiva, sarebbero ormai "correntemente usati" in ambito gastronomico. Qualcuno esprime a proposito di serratezza il dubbio che in realtà sia "una pura invenzione, al pari di scioglievolezza"; infine c'è chi trova "deprecabile" l'aggettivo sporchevole.
\end{abstract}

\section{Scioglievolezza, serratezza, sporchevole: dubbi scioglibili o scioglievoli? Speriamo di riuscire a scioglierli!}

La scioglievolezza è ormai una caratteristica che tutti associamo a un famoso cioccolatino grazie a un'azzeccata trovata pubblicitaria databile negli ultimi anni 'go del Novecento. Il primo spot reperibile in rete (Youtube) è del 1998 e le prime attestazioni scritte sono, con molta probabilità, gli inserti pubblicitari usciti sul quotidiano "La Stampa" del 5 e 6 novembre 1999 che, a mezza pagina, annunciavano: "Ti aspettiamo Sabato 6 novembre in pasticceria e nei migliori negozi specializzati per gustare l'incredibile scioglievolezza di ...". Una parola nuova che senza dubbio ha funzionato ed è riuscita a ritagliarsi un piccolo, ma sempre crescente, spazio di utilizzo e di diffusione. Possiamo individuare più fattori che hanno contribuito al suo successo:

- è una parola formata secondo le regole di derivazione dell'italiano e quindi si è inserita senza scossoni nel paradigma di molti altri derivati simili (colpevolezza, gradevolezza, piacevolezza, scorrevolezza);

- colma perfettamente la mancanza di un sostantivo astratto che indichi la prerogativa del cioccolato, ma poi vedremo anche di molti altri alimenti, di sciogliersi piacevolmente in bocca;

- il dilagare dell'interesse per la gastronomia e la grande attenzione rivolta al cibo in questi ultimi anni ne hanno favorito l'impiego con riferimento ad alimenti e preparazioni diverse dal cioccolato.

Vediamo di approfondire ciascuno di questi aspetti. Per quel che riguarda la formazione della parola (cfr. Grossmann-Rainer 2004) si parte dal verbo sciogliere con un primo passaggio all'aggettivo scioglievole (con aggiunta del suffisso -evole), anch'esso non attestato nei dizionari, ma esistente "in potenza" poiché dobbiamo presupporlo come base per l'ulteriore aggiunta del suffisso -ezza. In realtà, fin dal Trecento è attestato in italiano un aggettivo derivato da sciogliere con l'aggiunta di un altro suffisso, -ibile: si tratta di scioglibile che, però, a parte la rarità delle sue occorrenze e la sua specializzazione per riferirsi a nodi, legami, clausole, mantiene un tratto modale nel significato che scioglievole non presenta. Scioglibile infatti significa 'che si può sciogliere' (molto più vicino a solubile, pensando al cibo), mentre scioglievole non contempla il tratto della possibilità, indicando piuttosto la caratteristica di sciogliersi facilmente (e sicuramente) e collocandosi più nettamente nella sfera della sensorialità. I due suffissi -evole e -ibile, rivali in italiano, hanno la stessa base etimologica con la differenza che -ibile ha seguito la trafila colta, direttamente dal latino, mentre -evole ha seguito la via popolare con le conseguenti trasformazioni fonologiche: contrariamente a quanto avvenuto in casi analoghi, il suffisso dotto -ibile è riuscito a imporsi su -evole che, nonostante la sua flessibilità dovuta all'iniziale vocalica (che si adatta alla base dei verbi di qualsiasi coniugazione), è meno affermato e, soprattutto, ha progressivamente esaurito la sua produttività. La maggior parte delle formazioni in -evole si attesta infatti nel 'zoo, con un altro picco nel '5oo, ma con drastico rallentamento nei secoli successivi (su questi suffissi si veda anche il recente contributo di Ilde Consales, Dal verbo all'aggettivo: note sugli aggettivi in -bile ed -evole, in Per la storia della formazione delle parole in italiano: un nuovo corpus in rete (midia) e nuove prospettive di studio, a cura di P. D'Achille, M. Grossmann, Firenze, Cesati, 2017, pp. II9-I44). Il GRADIT registra I5 derivati con-evole nel Novecento (unico marcato come comune: solletichevole), i7 nell'Ottocento (unici usuali ammirevole, deplorevole, riprovevole e valevole), in nel Settecento (oggi ancora comuni solo cedevole e considerevole); il GDLI con attestazione novecentesca segnala solo sporchevole (su cui torneremo) e vomitevole. Tale marginalizzazione rispetto a -ibile ha prodotto in -evole anche, in molti casi, l'indebolimento dei tratti modali, mantenuti ad esempio in pieghevole 'che si può piegare', cedevole 'che può cedere', 
ma spariti in valevole 'che vale', scorrevole 'che scorre', stucchevole 'che stucca', e in molti altri. I due suffissi si oppongono anche nella derivazione ulteriore da aggettivo a sostantivo astratto: -evole seleziona solo -ezza (amorevole > amorevolezza, autorevole $>$ autorevolezza, colpevole $>$ colpevolezza), mentre -ibile seleziona -itá (compatibile > compatibilitá, comprensibile > comprensibilità, leggibile > leggibilitá).

Da queste considerazioni appare come scioglievolezza vada a colmare uno spazio di significato non del tutto coperto né dal rarissimo scioglibilità né dal suo sinonimo solubilità, riferiti comunemente alla proprietà di una sostanza di sciogliersi in un liquido creando una soluzione.

Al successo e al dilagare della parola oltre i confini del linguaggio pubblicitario ha contribuito, in modo direi determinante, quell "italiano gastronomico", in sovraesposizione negli ultimi anni nella comunicazione di massa, che ha risvegliato (e talvolta indotto) un rinnovato e pervasivo interesse per l'arte culinaria, in tutte le sue possibili declinazioni, inventando e diffondendo forme e parole che richiamano esperienze sensoriali ed estetiche. In questo contesto scioglievolezza ha confermato la sua forza allusiva e simbolica e si è conquistata spazi ulteriori per qualificare cibi diversi dal cioccolato che offrono però la stessa piacevolezza di sciogliersi a contatto col palato. Confermano questa evoluzione le occorrenze in rete (I99.0oo per scioglievolezza e II2.0oo per scioglievole con Google Italia al 25/or/2019), che offrono un quadro in cui scioglievole e scioglievolezza ricorrono soprattutto in pasticceria, ma anche riferiti a cibi salati: scioglievolezza della mozzarella, del grasso, del filetto, del salmone, addirittura del polpo e poi troviamo la pizza scioglievole e l'impasto scioglievole.

Decisamente meno numerose, ma significative, le attestazioni sui principali giornali nazionali: oltre alla prima comparsa sulla "Stampa" del 1999 già citata in apertura, dall'archivio on line del quotidiano "la Repubblica" abbiamo (al o4/o2/2019) 6 occorrenze, la prima del 20I2, poi una del 2014 e quattro del 20I8. Dal 2014 la scioglievolezza appare in contesti in cui non si parla di cioccolato:

un gorgo di riso che vi porta inevitabilmente alla scioglievolezza infinita di una tenera finanziera posta al centro del piatto (31 maggio 2014);

la Crocchettina è una pizza ricca e opulenta, cornicione ripieno di ricotta, fior di latte e mortadella sul disco, crema di pistacchio a crudo insieme a croccanti bocconi di crocchetta di patate. Non appesantisce, riempie di gola e denota una mortadella di ottima scelta nel suo non cedere il passo al calore, ma esaltarsi nella scioglievolezza del grasso (27 marzo 2018);

polpette di alici [...]. Golose, al primo morso sorprendono per la consistenza, la scioglievolezza perfetta e il divertimento (22 giugno 2018);

[pizze] Buona digeribilità, leggerezza medio-alta - da considerare in una cena degustazione con una proposta di quattro ricette -, scioglievolezza al palato soddisfacente (I2 luglio 2018);

1a manzetta prussiana con la sua inconfondibile scioglievolezza, la pregiata fassona piemontese e la fiorentina chianina dell'omonima valle toscana (4 maggio 2018).

Solo nell'articolo del 20 I2 il termine è inserito in un contesto in cui si parla sì del cioccolato, ma in una drammatica denuncia che oppone la scioglievolezza degli scintillanti cioccolatini protagonisti delle nostre feste, a quella degli occhi inondati di pianto dei bambini sfruttati nelle piantagioni di cacao:

Che mondo sarebbe senza bambini schiavi venduti a 230 euro l'uno? E quanta scioglievolezza c'è nel pianto silenzioso di un undicenne abbandonato alla frontiera e pronto per essere caricato dal primo trafficante che passa? E ancora, quanta carica di energia servirà allo stesso fanciullo prima che diventi forte e robusto, tanto da sfuggire ai suoi aguzzini senza essere centrato da una fucilata? C'è un angolo della Terra dove il cioccolato non fa male ai denti dei bambini, ma alla loro schiena. Un triangolo, per l'esattezza, che comprende i poverissimi stati del Mali e del Burkina Faso confinanti con la Costa d'Avorio, il Paese che produce il 40 per cento di tutto il cacao consumato nel mondo ("La Repubblica", I2 ottobre 20I2).

L'archivio del "Corriere della Sera" restituisce solo 2 occorrenze: in un articolo del 2r novembre 2009 Il bove del Carducci che sfida i vegetariani in cui la scioglievolezza è riferita alla carne, e in un altro del zo gennaio 2015 intitolato Piccoli ma pieni di qualsiasi cosa. Viaggio nell'Italia dei panini, in cui la scioglievolezza è caratteristica degli ingredienti che servono a farcire panini sempre più fantasiosi e imprevedibili.

Serratezza presenta lo stesso suffisso -ezza, ma aggiunto a un aggettivo qualificativo (in questo caso a un participio 
passato) in -to (serrato), secondo la stessa regola derivativa, mediamente produttiva, per cui in italiano si hanno, ad esempio, asciutto>asciuttezza, assoluto>assolutezza, moderato>moderatezza, trascurato>trascuratezza, ecc. Nella maggioranza dei casi il suffisso seleziona aggettivi o participi lessicalizzati che esprimono disposizioni umane (pacatezza, raffinatezza, spietatezza, ecc.), ma esiste anche un gruppo di aggettivi in -ezza "specializzati", potremmo dire, per esprimere un giudizio estetico: elaboratezza, ornatezza, stringatezza (ma anche larghezza, ampiezza, pienezza per esempio riferite a un suono). Possiamo far rientrare in questo ambito ristretto anche serratezza, attestato nel GDLI con le definizioni di 'rapidità, concisione di stile' (usato da Croce) e di 'chiusura, cupezza del volto' (con esempio tratto da Fenoglio) e nel GRADIT come parola comune e con definizioni analoghe ('lessere serrato', 'rapidità, concisione di stile'). Dobbiamo però aggiungere che si tratta di un sostantivo decisamente poco ricorrente (anche in rete, al 25/or/2019 se ne traggono solo 607 risultati), che rimane circoscritto all'ambito della terminologia della stilistica, in contesti del tipo serratezza della composizione, della scrittura, dello sviluppo drammatico, del racconto, delle argomentazioni, del discorso, narrativa, del romanzo nero, ecc. Non si tratta quindi di una parola nuova e nemmeno di una parola inesistente, ma di un termine molto raro e di impiego fortemente limitato.

La storia di sporchevole, altro aggettivo in -evole sulla base del verbo sporcare, è tutta novecentesca e il GDLI lo registra tra i nuovi aggettivi in -evole del secolo accanto solo a vomitevole. Lo registrano anche i maggiori dizionari sincronici (Treccani on line, DISC, GRADIT, Garzanti on line, Hoepli on line) indicandolo come non comune o scherzoso, e annotando due accezioni; (I) 'che puo sporcare, insudiciare, imbrattare'; (2) 'che ha facilità di sporcarsi', riferito spesso a tessuti. Non è invece attestato sporcabile (derivato possibile con il suffisso -abile, alternativo a -evole) che, in ogni caso, non potrebbe prevedere il tratto semantico attivo di 'qualcosa che può sporcare', ma solo quello passivo di 'qualcosa che puo essere sporcato'.

La prima attestazione di sporchevole offerta dal GDLI rimanda a Bruno Migliorini (in Parole nuove, I963) e a un articolo uscito sul "Corriere d'informazione" il $15 / 06 / 1948$ ("I tifosi... persino per andare a letto indossano un pigiama a righe neroazzurre e lo cambiano ogni due settimane perché quei colori sono poco sporchevoli”), ma una ricerca sugli archivi digitali dei quotidiani ha fatto emergere unoccorrenza dell'aggettivo, proprio riferito a un tessuto, già in un articolo uscito sul "Corriere della Sera" del I7 marzo 1934 intitolato Primo sguardo sulla cittá giapponese (Osaka), in cui si legge:

«Mogà» e «Mobò». Quanto durerà? Le ragazzine che vanno a scuola, le impiegate, le commesse hanno dovuto rinnegare il kimono. È troppo ingombrante, complicato e sporchevole. La maggior parte di queste seguaci della moda occidentale sono delle mogà (neologismo giapponese, da modern girl, cui corrisponde nell'altro sesso mobó, modern boy) per forza.

In effetti, una delle collocazioni più ricorrenti di sporchevole è proprio quella con i tessuti e i colori che hanno la prerogativa di sporcarsi (o non sporcarsi) facilmente: tra queste colpisce particolarmente la pubblicità, uscita il 4 marzo 1958 sul quotidiano "La Stampa" dell'impermeabile in terital "ingualcibile e non sporchevole", simbolo di progresso e innovazione dell'industria tessile italiana (l'immagine si può vedere consultando l'archivio storico della "Stampa" qui).

Viene la tentazione di far risalire a quel terital, tessuto simbolo di modernità, le 4I4 occorrenze delle attuali tovaglie meno sporchevoli (resinate e antimacchia) che si rintracciano in rete (Google Italia, 25/or/20r9), quelle fatte di materiale misto di stoffa e resine protettive.

Un altro ambito in cui si trovano sporadiche occorrenze di sporchevole/non sporchevole è, ancora una volta, quello culinario, per cui si possono classificare i cibi come più o meno sporchevoli a seconda della presenza o meno di sughi, salse e ingredienti liquidi che facilmente possono versarsi e sporcare chi sta mangiando. La prima attestazione in un contesto di questo genere è attribuita a Umberto Eco che, in Come mangiare in aereo (1987) contenuto nel Secondo Diario minimo (Bompiani, 1992), scriveva:

il buon senso vorrebbe che [in aereo] si servissero cibi non sporchevoli e compatti. [...] Cibi sporchevoli sono gli spaghetti con la pummarola 'n coppa, le melanzane alla parmigiana, le pizze appena uscite dal forno, il consommé in tazza bollente senza manici.

In rete (Google Italia 25/or/Io), dove complessivamente si rintracciano 15.600 occorrenze per sporchevole e 8.800 per sporchevoli, emerge un'altra recente collocazione in cui l'aggettivo è tornato in auge, quello con attivitá e lavori: nello specifico si hanno 729 risultati di attività sporchevoli in descrizioni di attività educative negli asili nido che 
prevedono la manipolazione di materiali (colori, impasti, cibi, ecc.) che sporcano e impiastricciano.

Per i giornali, oltre al "Corriere della Sera" e alla "Stampa" che, come abbiamo detto, ci hanno fornito attestazioni molto significative (anche se una soltanto per ciascuno) per precocità e specificità d'uso, "la Repubblica" conserva nel suo archivio complessivamente ro occorrenze (3 per sporchevole e 7 per il plurale sporchevoli): anche qui la più lontana nel tempo (comunque molto più recente delle altre) vede l'aggettivo appaiato con un indumento:

Lui e lei in camicie da notte bianche, da fantasmi nel castello, però molto 'sporchevoli' per il job. E le loro stanze sono in un seminterrato (da cui emergono come pantegane) perché i muri e le aperture del cortile sono fatti così (Macbeth nel nord est, 8 settembre 1996).

Nelle altre, databili tra il 2003 e il 20I8, troviamo l'aggettivo riferito a un "pavimento in gomma nera [...] non sporchevole"; alla carta carbone definita "la sporchevole regina degli uffici"; all'impastare "che tutti i bambini adorano perché è sporchevole", a mansioni di lavoro sporchevoli e ad attività sporchevoli per bambini.

In questo caso ci troviamo quindi di fronte a una parola esistente, attestata sui dizionari e ancora vitale, almeno in ambiti circoscritti e connotati spesso da un registro scherzoso e domestico, che si può quindi utilizzare tranquillamente.

\author{
Cita come: \\ Raffaella Setti, Scioglievolezza, serratezza, sporchevole: dubbi scioglibili o scioglievoli? Speriamo di \\ riuscire a scioglierli! , "Italiano digitale”, 2019, VIII, 2019/1 (gennaio-marzo) , pp. 46-49. \\ DOI: $10.35948 / 2532-9006 / 2019.3078$ \\ Copyright 2019 Accademia della Crusca \\ Pubblicato con licenza creative commons CC BY-NC-ND
}

